\title{
UPPER BOUNDS FOR THE MEANS OF EIGENVALUES OF RANDOM BOUNDARY VALUE PROBLEMS WITH WEAKLY CORRELATED COEFFICIENTS*
}

\author{
BY \\ WILLIAM E. BOYCE (Rensselaer Polytechnic Institute) \\ AND \\ NING - MAO XIA ( East China Institute of Chemical Technology, Shanghai)
}

\begin{abstract}
This paper concerns eigenvalue problems for second-order random differential equations with weakly correlated coefficients. The random problem and the mean (deterministic) problem are embedded in a parametrized problem whose eigenvalues are expanded in a power series in the parameter. This expansion leads, via the variational characterization of the eigenvalues, to computationally accessible upper bounds for the mean values of the eigenvalues of the original problem.
\end{abstract}

1. Introduction. Despite their significance for applications, random eigenvalue problems have received relatively little attention in comparison to that devoted to random initial value problems. When the random terms are small, perturbation methods have proved useful $[1,7,8,10]$. If, in addition, the random terms are weakly correlated, then in a certain limiting sense, each eigenvalue has nearly a normal distribution $[3,9,11]$. Variational methods have also found limited use [1,2]. In this paper we consider problems in which the random terms are not small so that a perturbation method is not appropriate. We seek to exploit the variational characterization of eigenvalues in order to obtain upper bounds for the mean of each eigenvalue of a fairly wide class of problems.

We consider the problem:

$$
\begin{aligned}
& -u^{\prime \prime}+q(x, \omega) u=\lambda u, \\
& u(0)=0, \quad u(1)=0
\end{aligned}
$$

where $q(x, \omega)$ is a random coefficient whose properties will be delineated later. It is helpful to introduce the more general problem

$$
\begin{gathered}
-u^{\prime \prime}+\left\{q_{0}(x)+\kappa\left[q(x, \omega)-q_{0}(x)\right]\right\} u=\lambda u, \\
u(0)=0, \quad u(1)=0
\end{gathered}
$$

\footnotetext{
${ }^{*}$ Received July 28,1983 . The results presented in this paper were obtained while the second author was a Visiting Scholar at Rensselaer Polytechnic Institute.
} 
indexed by the parameter $\kappa$, where $q_{0}(x)=\langle q(x, \omega)\rangle$ and $\langle\cdot\rangle$ denotes the mathematical expectation. When $\kappa=1$ the problem (1-2) reduces to (1-1), while when $\kappa=0$ it reduces to the deterministic problem

$$
\begin{gathered}
-u^{\prime \prime}+q_{0}(x) u=\lambda u, \\
u(0)=0, \quad u(1)=0 .
\end{gathered}
$$

Thus the parametrized problem (1-2) provides a means of relating (1-1) with (1-3).

To emphasize the fact that eigenvalues and eigenfunctions of (1-2) depend on $\kappa$ we denote them by $\lambda(\kappa)$ and $u(x, \kappa)$ respectively. They are analytic functions of $\kappa$ since $\kappa$ enters the problem (1-2) in an analytic manner. Thus we can write

$$
\lambda(\kappa)=\sum_{n=0}^{\infty} \frac{\lambda^{(n)}(0)}{n !} \kappa^{n}, \quad u(x, \kappa)=\sum_{n=0}^{\infty} \frac{u^{(n)}(x, 0)}{n !} \kappa^{n},
$$

and we assume that the radius of convergence of each of these series is at least one.

In Sec. 2 we discuss the calculation of $\lambda^{(n)}(0)$ and $u^{(n)}(x, 0)$ so that (1-4) can be used to determine $\lambda(\kappa)$ and $u(x, \kappa)$.

In Sec. 3 we assume that $q(x, \omega)-q_{0}(x)$ is weakly correlated with small correlation length $\varepsilon$. Then it follows that $\left\langle\lambda^{(2 n)}(0)\right\rangle=O\left(\varepsilon^{n}\right)$ and $\left\langle\lambda^{(2 n+1)}(0)\right\rangle=O\left(\varepsilon^{n+1}\right)$. This enables us to calculate $\langle\lambda(\kappa)\rangle$ and $\langle u(x, \kappa)\rangle$ from the finite sums

$$
\langle\lambda(\kappa)\rangle \cong \sum_{n=0}^{N} \frac{\left\langle\lambda^{(n)}(0)\right\rangle}{n !} \kappa^{n}, \quad\langle u(x, \kappa)\rangle \cong \sum_{n=0}^{N} \frac{\left\langle u^{(n)}(x, 0)\right\rangle}{n !} \kappa^{n}
$$

with an estimate of the error in terms of $\varepsilon$.

In using the formulas (1-5) we find that the coefficients $\left\langle\lambda^{(n)}(0)\right\rangle$ and $\left\langle u^{(n)}(x, 0)\right\rangle$ can be evaluated in terms of infinite eigenfunction expansions. If these are truncated after some finite number of terms, then an approximation for $\langle\lambda(\kappa)\rangle$ is obtained, which can be improved by using more terms in the eigenfunction series. The approximations for $\left\langle\lambda^{(n)}(0)\right\rangle$ and $\left\langle u^{(n)}(x, 0)\right\rangle$ can be related to a finite-dimensional (matrix) eigenvalue problem, whose eigenvalues are upper bounds for the eigenvalues of the boundary problem. This is discussed in Sec. 4, and an example is given in Sec. 5.

The methods presented in this paper, while largely formal, are among the first to bring variational principles and techniques to bear on random eigenvalue problems in a potentially effective way. Further, these methods can be extended to problems other than (1-1) in a straightforward way. Since many other second-order equations can be reduced to the form (1-1a) by a preliminary transformation, the problem (1-1) is already fairly general. However, other second-order equations, higher-order equations, or other boundary conditions, can be handled as discussed here. In any event the problem must be self-adjoint, and the eigenvalues bounded below.

2. Eigenvalues and eigenfunctions of the parametrized problem. To use the series expansions (1-4) for $\lambda(\kappa)$ and $u(x, \kappa)$ it is necessary to derive expressions for derivatives of $\lambda$ and $u$ with respect to $\kappa$; we denote these derivatives by $\lambda^{(n)}(\kappa)$ and $u^{(n)}(x, \kappa)$, respectively, for $n=1,2, \ldots$. 
The first step is to determine a boundary value problem satisfied by $u^{(n)}$. Next we describe an iterative process for the calculation of successive derivatives of any eigenvalue $\lambda_{l}(\kappa)$ and eigenfunction $u_{l}(x, \kappa)$ of (1-2). We also establish that this process can be continued indefinitely so that in principle, at least, all derivatives of $\lambda_{l}(\kappa)$ and $u_{l}(x, \kappa)$ can be found. Finally, explicit formulas are given for $\lambda_{l}^{(n)}(\kappa)$ and $u_{l}^{(n)}(x, \kappa)$ for any $n$.

By differentiating (1-2a) $n$ times with respect to $\kappa$ we obtain

$$
-u^{(n)}+q_{0} u^{(n)}=Q^{(n)}(x, \kappa),
$$

where

and

$$
Q(x, \kappa)=\lambda u-\kappa\left[q(x, \omega)-q_{0}(x)\right] u
$$

$$
Q^{(n)}(x, \kappa)=\partial^{n} Q(x, \kappa) / \partial \kappa^{n}
$$

It is possible to show that

$$
Q^{(n)}(x, \kappa)=\left[\lambda u^{(n)}-\kappa\left(q-q_{0}\right) u^{(n)}\right]+p_{n}
$$

where

$$
p_{n}(x, \kappa)=\sum_{j=1}^{n} C_{j}^{n} \lambda^{(j)}(\kappa) u^{(n-j)}(x, \kappa)-n\left[q(x, \omega)-q_{0}(x)\right] u^{(n-1)}(x, \kappa),
$$

and $C_{j}^{n}$ are binomial coefficients. Thus (2-1) has the form

$$
-u^{(n)^{\prime \prime}}+\left[q_{0}+\kappa\left(q-q_{0}\right)\right] u^{(n)}-\lambda u^{(n)}=p_{n}, \quad n=1,2, \ldots
$$

which is similar to (1-2a) except for the term $p_{n}$. The boundary conditions for $u^{(n)}$ follow directly from (1-2b), namely:

$$
u^{(n)}(0, \kappa)=0, \quad u^{(n)}(1, \kappa)=0 .
$$

If we let $\lambda_{l}(\kappa)$ and $u_{l}(x, \kappa)$ be the $l$ th eigenvalue and eigenfunction of $(1-2 \mathrm{a}, \mathrm{b})$ and assume that $u_{l}$ satisfies the normalization condition

$$
\int_{0}^{1} u_{l}^{2}(x, \kappa) d x=1,
$$

then it is easy to show that

$$
\lambda_{l}^{(1)}(\kappa)=\int_{0}^{1}\left[q(x, \omega)-q_{0}(x)\right] u_{l}^{2}(x, \kappa) d x .
$$

This is accomplished by replacing $u$ and $\lambda$ in (1-2) by $u_{l}$ and $\lambda_{l}$ respectively, differentiating (1-2a) once with respect to $\kappa$, multiplying the resulting equation by $u_{l}$, and integrating over $[0,1]$. Integrating by parts and making use of (1-2a) then yields (2-6). Higher derivatives of $\lambda_{l}(\kappa)$ are then given by:

$$
\begin{aligned}
& \lambda_{l}^{(n)}(\kappa)=\left\{\int_{0}^{1}\left[q(x, \omega)-q_{0}(x)\right] u_{l}^{2}(x, \kappa) d x\right\}^{(n-1)} \\
&=\int_{0}^{1}\left[q(x, \omega)-q_{0}(x)\right] \frac{d^{n-1}}{d \kappa^{n-1}}\left[u_{l}(x, \kappa) u_{l}(x, \kappa)\right] d x \\
&=\sum_{j=0}^{n-1} C_{j}^{n-1} \int_{0}^{1}\left[q(x, \omega)-q_{0}(x)\right] u_{l}^{(j)}(x, \kappa) u_{l}^{(n-1-j)}(x, \kappa) d x \\
& n=2,3, \ldots
\end{aligned}
$$


To determine the successive derivatives of $\lambda_{l}(\kappa)$ and $u_{l}(x, \kappa)$ with respect to $\kappa$ we start by solving (1-2) and then use (2-6) to find $\lambda_{l}^{(1)}(\kappa)$. Next we find $p_{1}(x, \kappa)$ from (2-3), then solve (2-4) with $n=1$ to find $u_{l}^{(1)}(x, \kappa)$, and finally find $\lambda_{l}^{(2)}(\kappa)$ from (2-7). Then the process is repeated: $p_{2}(x, \kappa), u_{l}^{(2)}(x, \kappa)$, and $\lambda_{l}^{(3)}(\kappa)$ are determined from (2-3), (2-4), and (2-7) respectively, and so on.

To guarantee that this procedure can be continued indefinitely we must show that

$$
\int_{0}^{1} p_{n l}(x, \kappa) u_{l}(x, \kappa) d x=0, \quad n=1,2, \ldots
$$

where $p_{n l}(x, \kappa)$ is obtained from (2-3) by replacing $\lambda$ and $u$ by $\lambda_{l}$ and $u_{l}$ respectively. For $n=1$,

$$
p_{1 l}=\lambda_{l}^{(1)} u_{l}-\left(q-q_{0}\right) u_{l}
$$

and it follows that

$$
\int_{0}^{1} p_{1 l} u_{l} d x=\lambda_{l}^{(1)} \int_{0}^{1} u_{l}^{2} d x-\int_{0}^{1}\left(q-q_{0}\right) u_{l}^{2} d x=0
$$

because of (2-5) and (2-6). An inductive argument can be used to establish (2-8) for $n=2,3, \ldots$.

Now let us consider the form of the solution of (2-4). Assume that $\lambda_{l}(\kappa)$ is never zero, and let $G(x, y, \kappa)$ be the Green's function for the operator

$$
L(\kappa)=-\frac{d^{2}}{d x^{2}}+\left\{q_{0}(x)+\kappa\left[q(x, \omega)-q_{0}(x)\right]\right\}
$$

with the boundary conditions $(2-4 \mathrm{~b})$. Then $u_{l}^{(n)}(x, \kappa)$ must satisfy the integral equation

$$
u_{l}^{(n)}(x, \kappa)-\lambda \int_{0}^{1} G(x, y, \kappa) u_{l}^{(n)}(y) d y=R_{n l}(x, \kappa),
$$

where

$$
R_{n l}(x, \kappa)=\int_{0}^{1} G(x, y, \kappa) p_{n l}(y, \kappa) d y,
$$

and $p_{n l}(x, \kappa)$ is given by (2-3) with $\lambda$ and $u$ replaced by $\lambda$, and $u$, respectively. It is straightforward to show that

$$
\begin{aligned}
\left(R_{n l}, u_{s}\right) & =\int_{0}^{1} R_{n l}(x, \kappa) u_{s}(x, \kappa) d x \\
& =\frac{1}{\lambda_{s}} \int_{0}^{1} p_{n l}(y, \kappa) u_{s}(y, \kappa) d s, \quad n=0,1,2, \ldots
\end{aligned}
$$

and that $\left(R_{n l}, u_{l}\right)=0$. Then, assuming that

$$
u_{l}^{(n)}(x, \kappa)=\sum_{s=1}^{\infty} a_{l s} u_{s}(x, \kappa), \quad R_{n l}(x, \kappa)=\sum_{s=1}^{\infty}\left(R_{n l}, u_{s}\right) u_{s}(x, \kappa),
$$

it follows that

$$
u_{l}^{(n)}(x, \kappa)=\sum_{\substack{s=1 \\ s \neq l}}^{\infty} \frac{\lambda_{s}(\kappa)}{\lambda_{s}(\kappa)-\lambda_{l}(\kappa)}\left(R_{n l}, u_{s}\right) u_{s}(x, \kappa)+C_{l n}(\kappa) u_{l}(x, \kappa),
$$


where the coefficient $C_{l n}(\kappa)$ is as yet unknown. To determine $C_{l n}$ form the inner product of $(2-16)$ with $u_{l}(x, \kappa)$, so that

$$
C_{l n}(\kappa)=\int_{0}^{1} u_{l}^{(n)}(x, \kappa) u_{l}(x, \kappa) d x
$$

Then differentiate

$$
\int_{0}^{1} u_{l}^{2}(x, \kappa) d x=1
$$

$n$ times with respect to $\kappa$; this gives

$$
\begin{aligned}
0= & \int_{0}^{1} \frac{d^{n}}{d \kappa^{n}} u_{l}^{2}(x, \kappa) d x=\int_{0}^{1} \sum_{j=0}^{n} C_{j}^{n} u_{l}^{(j)}(x, \kappa) u_{l}^{(n-j)}(x, \kappa) d x \\
= & 2 \int_{0}^{1} u_{l}(x, \kappa) u_{l}^{(n)}(x, \kappa) d x \\
& +\sum_{j=1}^{n-1} C_{j}^{n} \int_{0}^{1} u_{l}^{(j)}(x, \kappa) u_{l}^{(n-j)}(x, \kappa) d x .
\end{aligned}
$$

Thus

$$
C_{l n}(\kappa)=\left\{\begin{array}{l}
-\frac{1}{2} \sum_{j=1}^{n-1} C_{j}^{n} \int_{0}^{1} u_{l}^{(j)}(x, \kappa) u_{l}^{(n-j)}(x, \kappa) d x, \quad n \geqslant 2 \\
0, \quad n=1
\end{array}\right.
$$

Finally, substituting from (2-14) and (2-20) into (2-16), we obtain an explicit expression for $u_{l}^{(n)}(x, \kappa)$, namely

$$
\begin{aligned}
u_{l}^{(n)}(x, \kappa)= & \sum_{\substack{s=1 \\
s \neq l}}^{\infty} \frac{u_{s}(x, \kappa)}{\lambda_{s}(\kappa)-\lambda_{l}(\kappa)}\left[\sum_{j=1}^{n} C_{j}^{n} \lambda_{l}^{(j)}(\kappa) \int_{0}^{1} u_{l}^{(n-j)}(y, \kappa) u_{s}(y, \kappa) d y\right. \\
& \left.\quad-n \int_{0}^{1}\left\{q(y, \omega)-q_{0}(y)\right\} u_{l}^{(n-1)}(y, \kappa) u_{s}(y, \kappa) d y\right] \\
- & -\frac{1}{2} u_{l}(x, \kappa) \sum_{j=1}^{n-1} C_{j}^{n} \int_{0}^{1} u_{l}^{(j)}(x, \kappa) u_{l}^{(n-j)}(x, \kappa) d x
\end{aligned}
$$

where the last term in (2-21) does not appear if $n=1$.

From (1-4) we have

$$
\left\langle\lambda_{l}(1)\right\rangle=\sum_{n=0}^{\infty} \frac{\left\langle\lambda_{l}^{(n)}(0)\right\rangle}{n !}
$$

and in principle we can obtain expressions for $\left\langle\lambda_{l}^{(n)}(0)\right\rangle$ from (2-6), (2-7), and (2-21). In practice, it is necessary to make further assumptions about the probabilistic nature of $q(x, \omega)$ in order for these expressions to be useful. In the next section we explore the consequences of assuming that $q(x, \omega)-q_{0}(x)$ is weakly correlated (in a certain precise 
sense). It ultimately follows from (2-22) that

$$
\left\langle\lambda_{l}(1)\right\rangle \leqslant \sum_{s=0}^{2 m} \frac{\left\langle\hat{\lambda}_{2 m, l}^{(s)}(0)\right\rangle}{s !}+O\left(\varepsilon^{m+1}\right),
$$

where $\hat{\lambda}_{2 m, l}^{(s)}(0)$ is obtained from (2-6), (2-7), and (2-21) by setting $u_{r}(x, 0)=0$ for $r \geqslant 2 m+1$. Inequality (2-23) is the main result of this paper; it is established in Sec. 4 by means of an argument based on the variational properties of eigenvalues.

3. Weakly correlated coefficients. The formulas (2-6), (2-7), and (2-21) developed in the preceding section can be evaluated at $\kappa=0$ to obtain $\lambda_{l}^{(n)}(0)$ and $u_{l}^{(n)}(x, 0)$. Then the power series expansions

$$
\begin{gathered}
\lambda_{l}(\kappa)=\sum_{n=0}^{\infty} \frac{\lambda_{l}^{(n)}(0)}{n !} \kappa^{n}, \\
u_{l}(x, \kappa)=\sum_{n=0}^{\infty} \frac{u_{l}^{(n)}(x, 0)}{n !} \kappa^{n},
\end{gathered}
$$

can be used to find the desired eigenvalue $\lambda_{l}(1)$ and eigenfunction $u_{l}(x, 1)$ of the original random eigenvalue problem (1-1). Unfortunately, in practice we can only evaluate finitely many of the terms in (3-1), so the question arises of estimating the errors made in truncating the series in (3-1).

To this end, it is useful to examine the structure of $\lambda_{l}^{(n)}(\kappa)$ and $u_{l}^{(n)}(x, \kappa)$ in more detail. When $\kappa=0$, as required in (3-1), we show that the randomness in $\lambda_{l}^{(n)}(0)$ and $u_{l}^{(n)}(x, 0)$ depends in a relatively simple way on $q(x, \omega)-q_{0}(x)$. This enables us, when $q(x, \omega)-$ $q_{0}(x)$ is weakly correlated, to derive the expressions (3-12) and (3-13) for $\left\langle\lambda_{l}(\kappa)\right\rangle$ and $\left\langle u_{l}(x, \kappa)\right\rangle$ with an order estimate of the remainder in terms of the correlation length $\varepsilon$.

First, it is possible to show that $\lambda_{l}^{(n)}(\kappa)$ and $u_{l}^{(n)}(x, \kappa)$ can be expressed in the form

$$
\begin{aligned}
\lambda_{l}^{(n)}(\kappa) & =\sum_{i_{1}, \ldots, j_{n}} C_{i_{1} \cdots j_{n}} I_{i_{1} j_{1}} I_{i_{2} j_{2}} \cdots I_{i_{n} j_{n}}, \\
u_{l}^{(n)}(x, \kappa) & =\sum_{s, i_{1}, \ldots, j_{n}} \tilde{C}_{i_{1} \cdots j_{n} s} I_{i_{\nu_{1}} j_{1}} I_{i_{2} j_{2}} \cdots I_{i_{n} j_{n}} u_{s}(x, \kappa),
\end{aligned}
$$

where

$$
I_{i_{k} j_{k}}(\kappa)=\int_{0}^{1}\left[q(x, \omega)-q_{0}(x)\right] u_{i_{k}}(x, \kappa) u_{j_{k}}(x, \kappa) d x, \quad k=1, \ldots, n .
$$

It is convenient to express $\lambda_{l}^{(n)}(\kappa)$ and $u_{l}^{(n)}(x, \kappa)$ in the form (3-2a, b) in part because when $\kappa=0$ the coefficients $C_{i_{1} \cdots j_{n}}$ and $\tilde{C}_{i_{1} \cdots j_{n} s}$ become deterministic constants. The results (3-2a, b) can be established by induction. For $n=1$ we have from (2-6) that

$$
\lambda_{l}^{(1)}(\kappa)=\int_{0}^{1}\left[q(x, \omega)-q_{0}(x)\right] u_{l}^{2}(x, \kappa) d x=I_{l l}(\kappa),
$$


which is of the form (3-2a) with $n=1, C_{l l}=1$, and $C_{i_{1} j_{1}}=0$ otherwise. Also, from (2-21) with $n=1$ we obtain

$$
u_{l}^{(1)}(x, \kappa)=-\sum_{\substack{s=1 \\ s \neq l}}^{\infty} \frac{1}{\lambda_{s}-\lambda_{l}} I_{l s}(\kappa) u_{s}(x, \kappa),
$$

which is of the form (3-2b) with $n=1, \tilde{C}_{l s s}=-\left(\lambda_{s}-\lambda_{l}\right)^{-1}$, and $\tilde{C}_{i_{\nu_{1} s}}=0$ otherwise.

To complete the induction proof we assume that (3-2a, b) hold for $n=1, \ldots, m$, and must show that (3-2a, b) also hold for $n=m+1$. To obtain (3-2a) we start by writing (2-7) in the form

$$
\lambda_{l}^{(m+1)}(\kappa)=\sum_{k=0}^{m} C_{k}^{m} \int_{0}^{1}\left[q(x, \omega)-q_{0}(x)\right] u_{l}^{(k)}(x, \kappa) u_{l}^{(m-k)}(x, \kappa) d x .
$$

Substituting for $u_{l}^{(k)}$ and $u_{l}^{(m-k)}$ from (3-2b), we obtain:

$$
\begin{aligned}
\lambda_{l}^{(m+1)}(\kappa)= & \sum_{k=0}^{m} C_{k}^{m} \int_{0}^{1}\left[q-q_{0}\right] \sum_{s_{1}, i_{1}, \ldots, j_{k}} \tilde{C}_{i_{1} \cdots j_{k} s_{1}} I_{i_{1} j_{1}} \cdots I_{i_{k} j_{k}} u_{s_{1}}(x, \kappa) \\
& \cdot \sum_{s_{2}, i_{1}, \ldots, j_{m-k}} \tilde{C}_{i_{1} \cdots j_{m}-k} I_{i_{2}} \cdots I_{i_{i_{m}-k j_{m}-k}} u_{s_{2}}(x, \kappa) d x \\
= & \sum_{k=0}^{m} C_{k}^{m} \sum_{\substack{s_{1}, s_{2}, i_{1}, \ldots, j_{m}}} \hat{C}_{i_{1} \cdots j_{m} s_{1} s_{2}} I_{i_{1} j_{1}} \cdots I_{i_{m} j_{m}} \\
& \cdot \int_{0}^{1}\left[q(x, \omega)-q_{0}(x)\right] u_{s_{1}}(x, \kappa) u_{s_{2}}(x, \kappa) d x \\
= & \sum_{k=0}^{m} C_{k}^{m} \sum_{i_{1}, \ldots, j_{m+1}} \hat{C}_{i_{1} \ldots, j_{m+1}} I_{i_{1_{1}}} \cdots I_{i_{m} j_{m}} I_{i_{m+1} j_{m+1}} \\
= & \sum_{i_{1}, \ldots, j_{m+1}} C_{i_{1}, \ldots, j_{m+1}}^{*} I_{i_{i_{1}} j_{1}} \cdots I_{i_{m+1} j_{m+1}}
\end{aligned}
$$

which establishes (3-2a) in general. The derivation of $(3-2 b)$ in the general case follows similar lines and will be omitted.

Our ultimate objective is to use (3-1) to estimate $\left\langle\lambda_{l}(\kappa)\right\rangle$ and $\left\langle u_{l}(x, \kappa)\right\rangle$, so we turn now to a consideration of the quantities $\lambda_{l}^{(n)}(0)$ and $u_{l}^{(n)}(x, 0)$, which are given by (3-2) with $\kappa=0$. In this case the coefficients $C_{i_{1} \cdots j_{n}}$ and $\tilde{C}_{i_{1} \cdots j_{n} s}$ in (3-2) are deterministic, and when $\kappa=0$ the function $u_{i}(x, 0)$ is also deterministic, since it is an eigenfunction of the deterministic eigenvalue problem (1-3). Thus randomness enters the expressions for $\lambda_{l}^{(n)}(0)$ and $u_{l}^{(n)}(x, 0)$ only through the factors $q(x, \omega)-q_{0}(x)$ which appear in $I_{i_{k} j_{k}}(0)$. From (3-2) and (3-3) we have

$$
\begin{array}{r}
\left\langle\lambda_{l}^{(n)}(0)\right\rangle=\sum_{i_{1}, \ldots, j_{n}} C_{i_{1} \cdots j_{n}} \int_{0}^{1} \cdots \int_{0}^{1}\left\langle\left[q\left(x_{1}, \omega\right)-q_{0}\left(x_{1}\right)\right] \cdots\left[q\left(x_{n}, \omega\right)-q_{0}\left(x_{n}\right)\right]\right\rangle \\
\cdot u_{i_{1}}\left(x_{1}, 0\right) u_{j_{1}}\left(x_{1}, 0\right) \cdots u_{i_{n}}\left(x_{n}, 0\right) u_{j_{n}}\left(x_{n}, 0\right) d x_{1} \cdots d x_{n},
\end{array}
$$

together with a similar expression for $\left\langle u_{l}^{(n)}(x, 0)\right\rangle$. 
In order to simplify the expression (3-6) we assume that $q(x, \omega)$ has a short correlation length $\varepsilon$. More precisely, we follow Purkert and vom Scheidt [9] and assume that $q(x, \omega)$ is weakly correlated in the following sense. Let $S=\left(x_{1}, \ldots, x_{n}\right)$ be an $n$-tuple of real numbers and let $\varepsilon$ be a small positive constant. Let $S_{1}=\left(x_{i_{1}}, \ldots, x_{i_{k}}\right)$ be a subset of $S$, and suppose that $x_{i_{1}} \leqslant x_{i_{2}} \leqslant \cdots \leqslant x_{i_{k}}$; this ordering can always be achieved by relabeling the elements of $S_{1}$ if necessary. Then $S_{1}$ is said to be $\varepsilon$-neighboring if

$$
\left|x_{i_{1}}-x_{i_{2}}\right| \leqslant \varepsilon,\left|x_{i_{2}}-x_{i_{3}}\right| \leqslant \varepsilon, \ldots,\left|x_{i_{k-1}}-x_{i_{k}}\right| \leqslant \varepsilon .
$$

A single element subset is always $\varepsilon$-neighboring. The subset $S_{1}$ is maximally $\varepsilon$-neighboring, with respect to $S$, if $S_{1}$ is $\varepsilon$-neighboring, but is not contained in any larger $\varepsilon$-neighboring subset of $S$. It can be shown that $S$ can be separated into disjoint maximally $\varepsilon$-neighboring subsets in a unique way [9]. Then a stochastic process $f(x, \omega)$ is said to be weakly correlated with correlation length $\varepsilon$ if, for each $n$,

$$
\begin{aligned}
& \left\langle f\left(x_{1}, \omega\right) \cdots f\left(x_{n}, \omega\right)\right\rangle \\
& \quad=\left\langle f\left(x_{11}, \omega\right) \cdots f\left(x_{1 p_{1}}, \omega\right)\right\rangle \cdots\left\langle f\left(x_{k 1}, \omega\right) \cdots f\left(x_{k p_{k}}, \omega\right)\right\rangle,
\end{aligned}
$$

where the $n$-tuple $S$ has been separated into the maximally $\varepsilon$-neighboring subsets $\left(x_{11}, \ldots, x_{1 p_{1}}\right), \ldots,\left(x_{k 1}, \ldots, x_{k p_{k}}\right)$ and $\sum_{i=1}^{k} p_{i}=n$.

Thus we assume that $q(x, \omega)$ has the property (3-8). In particular, if $n=2$, then (3-8) reduces to

$$
\left\langle\left[q\left(x_{1}, \omega\right)-q_{0}\left(x_{1}\right)\right]\left[q\left(x_{2}, \omega\right)-q_{0}\left(x_{2}\right)\right]\right\rangle=\left\{\begin{array}{cc}
0, & \left|x_{1}-x_{2}\right|>\varepsilon \\
R_{q}\left(x_{1}, x_{2}\right), & \left|x_{1}-x_{2}\right| \leqslant \varepsilon .
\end{array}\right.
$$

If $q(x, \omega)$ is also stationary, then $R_{q}\left(x_{1}, x_{2}\right)$ has the form

$$
R_{q}\left(x_{1}, x_{2}\right)=\sigma_{q}^{2} \rho_{q}\left(x_{2}-x_{1}\right),
$$

where $\sigma_{q}^{2}$ is the (constant) variance and $\rho_{q}$ is the autocorrelation function of the process $q(x, \omega)-q_{0}(x)$. Equation (3-9) says that $q\left(x_{1}, \omega\right)-q_{0}\left(x_{1}\right)$ and $q\left(x_{2}, \omega\right)-q_{0}\left(x_{2}\right)$ are uncorrelated except in a strip of width $\sqrt{2} \varepsilon$ about the line $x_{2}=x_{1}$.

Using straightforward arguments similar to those in [3], it is possible to show that

$$
\begin{aligned}
\left\langle\lambda_{l}^{(2 m)}(0)\right\rangle & =O\left(\varepsilon^{m}\right), \quad\left\langle u_{l}^{(2 m)}(x, 0)\right\rangle=O\left(\varepsilon^{m}\right), \\
\left\langle\lambda_{l}^{(2 m+1)}(0)\right\rangle & =O\left(\varepsilon^{m+1}\right), \quad\left\langle u_{l}^{(2 m+1)}(x, 0)\right\rangle=O\left(\varepsilon^{m+1}\right) .
\end{aligned}
$$

These estimates for $\left\langle\lambda_{l}^{(n)}(0)\right\rangle$, for example, come from an identification of the contributions to (3-6) that are of lowest order in $\varepsilon$.

We can now use (3-11) in conjunction with (3-1) to estimate $\left\langle\lambda_{l}(\kappa)\right\rangle$ to any given order in $\varepsilon$. For instance, if we wish to keep terms up to order $\varepsilon^{m}$, we have

$$
\left\langle\lambda_{l}(\kappa)\right\rangle=\sum_{n=0}^{2 m} \frac{\left\langle\lambda_{l}^{(n)}(0)\right\rangle}{n !} \kappa^{n}+O\left(\varepsilon^{m+1}\right) .
$$


In a similar way

$$
\left\langle u_{l}(x, \kappa)\right\rangle=\sum_{n=0}^{2 m} \frac{\left\langle u_{l}^{(n)}(x, 0)\right\rangle}{n !} \kappa^{n}+O\left(\varepsilon^{m+1}\right) .
$$

4. Upper bounds for $\langle\lambda(\mathbf{1})\rangle$. For deterministic eigenvalue problems analogous to (1-1) it is often convenient to determine upper bounds for the respective eigenvalues using the method of Rayleigh-Ritz-Galerkin. This method [4, 5, 6] is based on the variational characterization of the eigenvalues when the eigenvalues are bounded below. To adapt this method to the problem under investigation here we start from the minimum principle corresponding to (1-1):

$$
\lambda(\omega)=\min \int_{0}^{1}\left[u^{\prime 2}(x, \omega)+q(x, \omega) u^{2}(x, \omega)\right] d x,
$$

where the minimization is carried out over an appropriate class of functions. For instance, to obtain $\lambda_{1}(\omega)$, we require that admissible functions satisfy $u(0, \omega)=u(1, \omega)=0$ and

$$
\int_{0}^{1} u^{2}(x, \omega) d x=1
$$

Since they automatically satisfy the proper boundary conditions, it is convenient to use the eigenfunctions of the corresponding deterministic problem (1-3) as basis functions. Thus we assume that

$$
u(x, \omega)=\sum_{i=1}^{n} c_{i}(\omega) u_{i}(x, 0),
$$

where the coefficients $c_{i}(\omega)$ are to be determined so as to minimize the right side of (4-1) subject to the condition (4-2). We continue to denote the $l$ th eigenvalue of (1-1) by $\lambda_{l}(1)$, since $(1-1)$ is obtained from (1-2) by setting $\kappa=1$. Then [4 (Page 224), for example] it follows that

$$
\lambda_{l}(1) \leqslant \Lambda_{n l}, \quad l=1, \ldots, n,
$$

where $\Lambda_{n l}$ is the $l$ th eigenvalue of the algebraic problem

$$
\mathbf{A} \boldsymbol{\xi}=\Lambda \xi,
$$

in which

$$
\boldsymbol{\xi}=\left(c_{1}, \ldots, c_{n}\right)^{T}, \quad \mathbf{A}=\left(A_{i j}\right),
$$

and

$$
\begin{array}{r}
A_{i j}(\omega)=\int_{0}^{1}\left[u_{i}^{\prime}(x, 0) u_{j}^{\prime}(x, 0)+q(x, \omega) u_{i}(x, 0) u_{j}(x, 0)\right] d x \\
i, j=1, \ldots, n .
\end{array}
$$

From (4-2) it follows that $\boldsymbol{\xi}^{T} \boldsymbol{\xi}=1$.

When $n=1,(4-4)$ reduces to

$$
\lambda_{1}(1) \leqslant \Lambda_{11}=A_{11}=\int_{0}^{1}\left[u_{1}^{\prime 2}(x, 0)+q(x, \omega) u_{1}^{2}(x, 0)\right] d x,
$$


whence

$$
\left\langle\lambda_{1}(1)\right\rangle \leqslant \lambda_{1}(0),
$$

a result that has been known [1] for some time. However, (4-9) does not readily generalize to higher eigenvalues [2].

To obtain improved estimates of $\lambda_{l}(1)$ from (4-4), we can let $n$ increase. Thus if $m \geqslant n \geqslant l$, we have

$$
\lambda_{l}(1) \leqslant \Lambda_{m l} \leqslant \Lambda_{n l} \leqslant \Lambda_{l l}
$$

and

$$
\lim _{m \rightarrow \infty} \Lambda_{m l}=\lambda_{l}(1) .
$$

In practice, the estimates (4-4) or (4-10) are not immediately very useful, because they require the determination of the eigenvalues of the $n \times n$ random matrix $\mathbf{A}$ whose elements are given by (4-7). However, we can also approach the algebraic eigenvalue problem (4-5) from the point of view of Secs. 2 and 3. Thus we separate the matrix $\mathbf{A}$ into two parts $\mathbf{B}=\left(B_{i j}\right)$ and $\mathbf{C}=\left(C_{i j}\right)$, where

$$
\begin{gathered}
B_{i j}=\int_{0}^{1}\left[u_{i}^{\prime}(x, 0) u_{j}^{\prime}(x, 0)+q_{0}(x) u_{i}(x, 0) u_{j}(x, 0)\right] d x, \\
C_{i j}=\int_{0}^{1}\left[q(x, \omega)-q_{0}(x)\right] u_{i}(x, 0) u_{j}(x, 0) d x ;
\end{gathered}
$$

then we can imbed (4-5) in the parametrized system:

$$
(\mathrm{B}+\kappa \mathrm{C}) \boldsymbol{\xi}=\Lambda \xi \text {. }
$$

The system (4-14) reduces to (4-5) when $\kappa=1$ and to the deterministic system

$$
\mathbf{B} \boldsymbol{\xi}=\Lambda \xi
$$

when $\kappa=0$. We denote the eigenvalues and eigenvectors of (4-14) by $\Lambda_{n l}(\kappa)$ and $\xi_{n l}(\kappa)$ respectively for $l=1, \ldots, n$. Observe that

$$
\Lambda_{n l}(0)=\lambda_{l}(0), \quad \xi_{n l}(0)=[0, \ldots, 1, \ldots, 0]^{T},
$$

where the number 1 appears in the $l$ th position in $\xi_{n l}(0)$.

We assume that $\Lambda_{n l}(\kappa)$ has the convergent power series expansion

$$
\Lambda_{n l}(\kappa)=\sum_{m=0}^{\infty} \frac{\Lambda_{n l}^{(m)}(0)}{m !} \kappa^{m},
$$

with radius of convergence $\rho>1$, where the superscript $(m)$ refers to the $m$ th derivative with respect to $\kappa$. As in Sec. 3, we now wish to determine the coefficients $\Lambda_{n l}^{(m)}(0)$. First we seek formulas for $\Lambda_{n l}^{(m)}(\kappa)$ similar to (2-6) and (2-7). Starting from

$$
(\mathbf{B}+\kappa \mathbf{C}) \xi_{n l}(\kappa)=\Lambda_{n l}(\kappa) \xi_{n l}(\kappa),
$$

we differentiate with respect to $\kappa$ to obtain

$$
\mathbf{C} \xi_{n l}(\kappa)+(\mathbf{B}+\kappa \mathbf{C}) \xi_{n l}^{\prime}(\kappa)=\Lambda_{n l}^{\prime}(\kappa) \xi_{n l}(\kappa)+\Lambda_{n l}(\kappa) \xi_{n l}^{\prime}(\kappa) .
$$

Multiplying (4-19) on the left by $\boldsymbol{\xi}_{n l}^{T}(\kappa)$ and using the symmetry of $\mathbf{B}$ and $\mathbf{C}$, we obtain

$$
\Lambda_{n l}^{(1)}(\kappa)=\xi_{n l}^{T}(\kappa) \mathbf{C} \xi_{n l}(\kappa),
$$


which is analogous to (2-6). Higher derivatives of $\Lambda_{n l}(\kappa)$ are obtained by repeated differentiation of (4-20), that is,

$$
\Lambda_{n l}^{(m)}(\kappa)=\sum_{q=0}^{m-1} C_{q}^{m-1} \boldsymbol{\xi}_{n l}^{(q)^{T}}(\kappa) \mathbf{C} \boldsymbol{\xi}_{n l}^{(m-1-q)}(\kappa),
$$

which is analogous to (2-7).

In order to use (4-20) and (4-21) to obtain $\Lambda_{n l}^{(m)}(0)$ we must first determine $\xi_{n l}^{(q)}(0)$ for $q=0,1, \ldots, m-1$. For $q=0$ we obtain $\xi_{n l}(0)$ by solving (4-15). Then, by differentiating (4-14) $q$ times we find that $\xi_{n}^{(q)}(\kappa)$ satisfies

$$
\left[\mathbf{B}+\kappa \mathbf{C}-\Lambda_{n l}(\kappa)\right] \boldsymbol{\xi}_{n l}^{(q)}(\kappa)=\mathbf{P}_{q n l}(\kappa), \quad q=1,2, \ldots,
$$

where

$$
\mathbf{P}_{q n l}(\kappa)=\sum_{j=1}^{q} C_{j}^{q} \Lambda_{n l}^{(j)}(\kappa) \boldsymbol{\xi}_{n l}^{(q-j)}(\kappa)-q \mathbf{C} \boldsymbol{\xi}_{n l}^{(q-1)}(\kappa), \quad q=1,2, \ldots
$$

When $\kappa=0(4-22)$ reduces to

$$
\left[\mathbf{B}-\Lambda_{n l}(0)\right] \boldsymbol{\xi}_{n l}^{(q)}(0)=\mathbf{P}_{q n l}(0),
$$

an equation that is readily solved. Multiplying (4-24) on the left by $\xi_{n s}^{T}(0)$, and using the symmetry of B and the fact that $\Lambda_{n s}(0)$ and $\xi_{n s}(0)$ satisfy (4-15), it follows that

$$
\boldsymbol{\xi}_{n l}^{(q)}(0) \boldsymbol{\xi}_{n s}(0)=\frac{\boldsymbol{\xi}_{n s}^{T}(0) \mathbf{P}_{q n l}(0)}{\Lambda_{n s}(0)-\Lambda_{n l}(0)}, \quad s \neq l .
$$

On the other hand, we can express $\xi_{n l}^{(q)}(0)$ as a linear combination of $\boldsymbol{\xi}_{n 1}(0), \ldots, \boldsymbol{\xi}_{n n}(0)$ :

$$
\begin{aligned}
\boldsymbol{\xi}_{n l}^{(q)}(0) & =\sum_{s=1}^{n}\left[\xi_{n l}^{(q)^{T}}(0) \boldsymbol{\xi}_{n s}(0)\right] \xi_{n s}(0) \\
& =\sum_{\substack{s=1 \\
s \neq l}}^{n} \frac{\xi_{n s}^{T}(0) \mathbf{P}_{q n l}(0)}{\Lambda_{n s}(0)-\Lambda_{n l}(0)} \xi_{n s}(0)+\left[\xi_{n l}^{(q)^{T}}(0) \xi_{n l}(0)\right] \xi_{n l}(0) .
\end{aligned}
$$

Finally, by differentiating the equation

$$
\xi_{n l}^{T}(\kappa) \xi_{n l}(\kappa)=1,
$$

we find that for $q=1$

$$
\xi_{n l}^{\prime T}(0) \xi_{n l}(0)=0
$$

and for $q \geqslant 2$

$$
\xi_{n l}^{(q)}(0) \xi_{n l}(0)=-\frac{1}{2} \sum_{j=1}^{q-1} C_{j}^{q \xi_{n l}^{(j)}}(0) \xi_{n l}^{(q-j)}(0)
$$


Thus (4-26) takes the form

$$
\begin{aligned}
\boldsymbol{\xi}_{n l}^{(q)}(0)= & \sum_{\substack{s=1 \\
s \neq l}}^{n} \frac{\boldsymbol{\xi}_{n s}^{T}(0) \mathbf{P}_{q n l}(0)}{\Lambda_{n s}(0)-\Lambda_{n l}(0)} \boldsymbol{\xi}_{n s}(0) \\
& -\frac{1}{2} \xi_{n l}(0) \sum_{j=1}^{q-1} C_{j}^{q \boldsymbol{\xi}_{n l}^{(j)}}(0) \boldsymbol{\xi}_{n l}^{(q-j)}(0), \quad q \geqslant 2,
\end{aligned}
$$

while for $q=1$ the last term on the right side of (4-30) is absent. By means of (4-20), (4-21), and (4-30), we can evaluate $\Lambda_{n l}^{(m)}(0)$, and then we can use (4-17) to determine $\Lambda_{n l}(\kappa)$ for any $\kappa$ in $0 \leqslant \kappa \leqslant 1$.

Next, we wish to consider the effect of reducing the number of base functions from $n$ to a number $r \leqslant n$. That is, we consider the base functions $v_{1}(x)=u_{1}(x, 0), \ldots, v_{r}(x)=$ $u_{r}(x, 0), v_{r+1}(x)=0, \ldots, v_{n}(x)=0$. We partition $\xi_{n l}(\kappa)$ so that

$$
\xi_{n l}(\kappa)=\left(\begin{array}{l}
\zeta_{r l}(\kappa) \\
\eta_{r l}(\kappa)
\end{array}\right)
$$

where $\zeta_{r l}(\kappa)$ includes the first $r$ components of $\zeta_{n l}(\kappa)$, and $\eta_{r l}(\kappa)$ contains the remaining $n-r$ components. The matrices $\mathbf{B}$ and $\mathbf{C}$ can be put in the form

$$
\mathbf{B}=\left(\begin{array}{cc}
\mathbf{B}_{11} & 0 \\
0 & 0
\end{array}\right), \quad \mathbf{C}=\left(\begin{array}{cc}
\mathbf{C}_{11} & 0 \\
0 & 0
\end{array}\right),
$$

where $\mathbf{B}_{11}$ and $\mathbf{C}_{11}$ are $r \times r$ submatrices. Then it is possible to show that

$$
\begin{aligned}
\boldsymbol{\xi}_{n l}^{(s)}(0) & =\left(\begin{array}{c}
\boldsymbol{\xi}_{r l}^{(s)}(0) \\
0
\end{array}\right), \\
\Lambda_{n l}^{(s)}(0) & =\Lambda_{r l}^{(s)}(0),
\end{aligned}
$$

for $l=1, \ldots, r$, where $1 \leqslant r \leqslant n$, and for $s=0,1,2, \ldots$, although these results are needed only for $s \leqslant r$.

For $s=0$ the results (4-33) are clearly true, and the general case can be handled by induction. Assume that (4-33a, b) are true for $q<s$ and then consider $q=s$. From (4-21) with $m=s$ and $\kappa=0$, note that

$$
\begin{aligned}
\Lambda_{n l}^{(s)}(0) & =\sum_{q=0}^{s-1} C_{q}^{s-1} \boldsymbol{\xi}_{n l}^{(q)}(0)\left(\begin{array}{cc}
\mathbf{C}_{11} & 0 \\
0 & 0
\end{array}\right) \boldsymbol{\xi}_{n l}^{(s-1-q)}(0) \\
& =\sum_{q=0}^{s-1} C_{q}^{s-1} \boldsymbol{\xi}_{r l}^{(q)}(0) \mathbf{C}_{11} \boldsymbol{\xi}_{r l}^{(s-1-q)}(0)=\Lambda_{r l}^{(s)}(0),
\end{aligned}
$$

which is (4-33b). Then substitute (4-31) and (4-32) in (4-22) and (4-23), and then set $\kappa=0$. By also making use of (4-33b) and (4-34) we obtain

$$
\begin{gathered}
\mathbf{B}_{11} \zeta_{r l}^{(s)}(0)-\Lambda_{r l}(0) \zeta_{r l}^{(s)}(0) \\
=\sum_{j=1}^{s} C_{j}^{s} \Lambda_{r l}^{(j)}(0) \zeta_{r l}^{(s-j)}(0)-s \mathbf{C}_{11} \zeta_{r l}^{(s-1)}(0), \\
\Lambda_{r l}(0) \eta_{r l}^{(s)}(0)=\mathbf{0} .
\end{gathered}
$$


Since $\Lambda_{r l}(0) \neq 0$, it follows from (4-35b) that $\eta_{r l}^{(s)}(0)=0$. Then from (4-35a) we have $\zeta_{r l}^{(s)}(0)=\xi_{r l}^{(s)}(0)$, and this establishes (4-33a).

Next, let us consider the relation between $\Lambda_{n l}^{(m)}(0)$ and $\lambda_{l}^{(m)}(0)$. The former is obtained by setting $\kappa=0$ in (4-20) or (4-21) while the latter is obtained in a similar way from (2-6) or (2-7). For $m=0$ it is evident that

$$
\Lambda_{n l}(0)=\lambda_{l}(0)
$$

Further, from (4-20) we have

$$
\begin{aligned}
\Lambda_{n l}^{(1)}(0) & =\xi_{n l}^{T}(0) \mathbf{C} \xi_{n l}(0)=C_{l l} \\
& =\int_{0}^{1}\left[q(x, \omega)-q_{0}(x)\right] u_{l}^{2}(x, 0) d x \\
& =\lambda_{l}^{(1)}(0), \quad l=1, \ldots, n,
\end{aligned}
$$

where (2-6) has been used in the last step.

The general case can be illustrated for $m=2$. In that case (4-21) yields

$$
\Lambda_{n l}^{(2)}(0)=\xi_{n l}^{T}(0) \mathbf{C} \xi_{n l}^{(1)}(0)+\xi_{n l}^{T^{(1)}}(0) C \xi_{n l}(0),
$$

where it follows from (4-30) that

$$
\xi_{n l}^{(1)}(0)=-\sum_{\substack{s=1 \\ s \neq l}}^{n} \frac{C_{s l}}{\Lambda_{n s}(0)-\Lambda_{n l}(0)} \xi_{n s}(0)
$$

with $C_{s l}$ given by (4-13). Then by (4-38)

$$
\Lambda_{n l}^{(2)}(0)=-2 \sum_{\substack{s=1 \\ s \neq l}}^{n} \frac{C_{s l}^{2}}{\Lambda_{n s}(0)-\Lambda_{n l}(0)}, \quad l=1, \ldots, n .
$$

On the other hand, from (2-7)

$$
\lambda_{l}^{(2)}(0)=2 \int_{0}^{1}\left[q(x, \omega)-q_{0}(x)\right] u_{l}(x, 0) u_{l}^{\prime}(x, 0) d x,
$$

where $u_{l}^{\prime}(x, 0)$ can be obtained from (2-21). Making this substitution, we obtain

$$
\lambda_{l}^{(2)}(0)=-2 \sum_{\substack{s=1 \\ s \neq l}}^{\infty} \frac{\left\{\int_{0}^{1}\left[q(x, \omega)-q_{0}(x)\right] u_{l}(x, 0) u_{s}(x, 0)\right\}^{2}}{\lambda_{s}(0)-\lambda_{l}(0)}
$$

On comparing the expressions for $\Lambda_{n l}^{(2)}(0)$ and $\lambda_{l}^{(2)}(0)$, we see that the right-hand side of (4-40) is identical with the first $n$ terms of the right side of (4-42). Thus

$$
\Lambda_{n l}^{(2)}(0)=\hat{\lambda}_{n l}^{(2)}(0) \text {, }
$$

where $\hat{\lambda}_{n l}^{(2)}(0)$ is obtained from $\lambda_{l}^{(2)}(0)$ by setting $u_{s}(x, 0)=0$ for $s=n+1, n+2, \ldots$ The above argument can be extended in a straightforward way and leads to the conclusion that

$$
\Lambda_{n l}^{(q)}(0)=\hat{\lambda}_{n l}^{(q)}(0)
$$

for all values of $q \geqslant 2$. Since (4-44) clearly holds for $q=0$ and $q=1$, this establishes (4-44) for all nonnegative integer values of $q$. 
Finally, we are in a position to estimate $\left\langle\lambda_{l}(1)\right\rangle$, the mean of the $l$ th eigenvalue of the original problem (1-1). From (4-4) and (4-17) we have

$$
\lambda_{l}(1) \leqslant \sum_{s=0}^{\infty} \frac{\Lambda_{n l}^{(s)}(0)}{s !}=\sum_{s=0}^{2 m} \frac{\Lambda_{n l}^{(s)}(0)}{s !}+\sum_{s=2 m+1}^{\infty} \frac{\Lambda_{n l}^{(s)}(0)}{s !},
$$

and therefore

$$
\left\langle\lambda_{l}(1)\right\rangle \leqslant \sum_{s=0}^{2 m} \frac{\left\langle\Lambda_{n l}^{(s)}(0)\right\rangle}{s !}+\sum_{s=2 m+1}^{\infty} \frac{\left\langle\Lambda_{n l}^{(s)}(0)\right\rangle}{s !} .
$$

As in Sec. 3 we know that

$$
\sum_{s=2 m+1}^{\infty} \frac{\left\langle\Lambda_{n l}^{(s)}(0)\right\rangle}{s !}=O\left(\varepsilon^{m+1}\right)
$$

and if we also choose $n=2 m$, then (4-46) becomes

$$
\left\langle\lambda_{l}(1)\right\rangle \leqslant \sum_{s=0}^{2 m} \frac{\left\langle\Lambda_{2 m, l}^{(s)}(0)\right\rangle}{s !}+O\left(\varepsilon^{m+1}\right) .
$$

To evaluate the right side of (4-48) we replace $\left\langle\Lambda_{2 m, l}^{(s)}(0)\right\rangle$ by $\left\langle\hat{\lambda}_{2 m, l}^{(s)}(0)\right\rangle$; this latter quantity can be calculated from $(2-6),(2-7)$, and $(2-21)$ by setting $u_{2 m+1}(x, 0)=$ $u_{2 m+2}(x, 0)=\cdots=0$.

5. Example. Consider the example

$$
\begin{aligned}
& -u^{\prime \prime}+q(x, \omega) u=\lambda u, \\
& u(0)=0, \quad u(1)=0
\end{aligned}
$$

where we assume that

$$
q_{0}(x)=\langle q(x, \omega)\rangle=0 .
$$

The corresponding deterministic problem is:

$$
\begin{gathered}
-u^{\prime \prime}-\lambda u \\
u(0)=0, \quad u(1)=0
\end{gathered}
$$

with the eigenvalues $\lambda_{l}=l^{2} \pi^{2}$ and eigenfucntions $u_{l}(x)=\sqrt{2} \sin l \pi x$. In terms of the parameter $\kappa$ the differential equation is

$$
-u^{\prime \prime}+\kappa q(x, \omega) u=\lambda u
$$

which reduces to $(5-3 \mathrm{a})$ or to $(5-1 \mathrm{a})$ if $\kappa=0$ or $\kappa=1$ respectively.

To estimate $\left\langle\lambda_{l}(1)\right\rangle$, that is, the mean of the $l$ th eigenvalue of $(5-1)$, up to terms of order $\varepsilon$, we make use of (4-48) with $m=1$. Thus

$$
\left\langle\lambda_{l}(1)\right\rangle \leqslant\left\langle\Lambda_{2 l}(0)\right\rangle+\left\langle\Lambda_{2 l}^{(1)}(0)\right\rangle+\frac{\left\langle\Lambda_{2 l}^{(2)}(0)\right\rangle}{2 !}+O\left(\varepsilon^{2}\right) .
$$

Further,

$$
\Lambda_{2 l}(0)=\lambda_{l}(0)=l^{2} \pi^{2}
$$


so

$$
\left\langle\Lambda_{2 l}(0)\right\rangle=l^{2} \pi^{2}
$$

Next,

$$
\Lambda_{2 l}^{(1)}(0)=\lambda_{l}^{(1)}(0)=\int_{0}^{1} q(x, \omega) u_{l}^{2}(x, 0) d x
$$

consequently

$$
\left\langle\Lambda_{2 l}^{(1)}(0)\right\rangle=\int_{0}^{1}\langle q(x, \omega)\rangle u_{l}^{2}(x, 0) d x=0
$$

Further

$$
\Lambda_{2 l}^{(2)}(0)=\hat{\lambda}_{2 l}^{(2)}(0)=-2 \sum_{\substack{s=1 \\ s \neq l}}^{2} \frac{\left[\int_{0}^{1} q(x, \omega) u_{l}(x, 0) u_{s}(x, 0) d x\right]^{2}}{\lambda_{s}(0)-\lambda_{l}(0)}
$$

Thus

$$
\begin{aligned}
& \left\langle\Lambda_{2 l}^{(2)}(0)\right\rangle \\
& \quad=-2 \sum_{\substack{s=1 \\
s \neq l}}^{2} \frac{\int_{0}^{1} \int_{0}^{1}\langle q(x, \omega) q(y, \omega)\rangle u_{l}(x, 0) u_{s}(x, 0) u_{l}(y, 0) u_{s}(y, 0) d x d y}{\lambda_{s}(0)-\lambda(0)} .
\end{aligned}
$$

The integral in (5-11) is simplified by (3-9), and the result is

$$
\left\langle\Lambda_{2 l}^{(2)}(0)\right\rangle=-2 \sum_{\substack{s=1 \\ s \neq l}}^{2} 2 \varepsilon \frac{\int_{0}^{1} R_{q}(z, z) u_{l}^{2}(z, 0) u_{s}^{2}(z, 0) d z}{\lambda_{s}(0)-\lambda_{l}(0)}+O\left(\varepsilon^{3}\right) ;
$$

the calculations are similar to those in [3] and need not be repeated here. Let us assume further that $R_{q}(z, z)=1$ and that $l=1$. Then $s=2$, and

$$
\int_{0}^{1} R_{q}(z, z) u_{l}^{2}(z, 0) u_{s}^{2}(z, 0) d z=4 \int_{0}^{1} \sin ^{2} \pi z \sin ^{2} 2 \pi z d z=1,
$$

so that

$$
\left\langle\Lambda_{21}^{(2)}(0)\right\rangle=-\frac{4 \varepsilon}{3 \pi^{2}}+O\left(\varepsilon^{3}\right) .
$$

Substituting (5-7), (5-9), and (5-14) into (5-5), we finally have

$$
\left\langle\lambda_{1}(1)\right\rangle \leqslant \pi^{2}-\frac{2 \varepsilon}{3 \pi^{2}}+O\left(\varepsilon^{2}\right) .
$$

Proceeding in the same way for $l=2$, we obtain

$$
\left\langle\lambda_{2}(1)\right\rangle \leqslant 4 \pi^{2}+\frac{2 \varepsilon}{3 \pi^{2}}+O\left(\varepsilon^{2}\right)
$$

Approximations that are higher order in $\varepsilon$ can be obtained from (4-48) in a similar manner, by using a larger value of $m$. To estimate $\left\langle\lambda_{l}(1)\right\rangle$ for $l>2$, we must also use a 
larger value of $m$, since we must always have $l \leqslant 2 m$. In these cases the calculations rapidly become much more cumbersome.

The expressions (5-15) and (5-16) are consistent with the results in [2], namely that while

$$
\left\langle\lambda_{1}(1)\right\rangle \leqslant \lambda_{1}(0)=\pi^{2},
$$

the corresponding inequality fails for the second and higher eigenvalues.

One can also obtain an estimate for $\left\langle\lambda_{l}(1)\right\rangle$ from the expression for the density function for $\lambda_{l}(1)$ given in [3]. However, this requires more detailed information about the random term $q(x, \omega)$ than does the method presented here.

\section{REFERENCES}

[1] W. E. Boyce, Random eigenvalue problems, in Probabilistic Methods in Applied Mathematics, vol. 1, A. T. Bharucha-Reid (editor), Academic Press, New York, 1968, 1-73

[2] W. E. Boyce, On a conjecture concerning the means of the eigenvalues of random Sturm-Liouville boundary value problems, Q. Appl. Math., 38, 241-245 (1980)

[3] W. E. Boyce and Ning-Mao Xia, The approach to normality of the solutions of random boundary and eigenvalue problems with weakly correlated coefficients, Q. Appl. Math., 40, 419-445 (1983)

[4] L. Collatz, Eigenwertprobleme und ihre numerische Behandlung, Chelsea Publishing Co., New York, 1948

[5] L. Collatz, Eigenwertaufgaben mit technischen Anwendungen, Geest and Portig, Leipzig, 1963

[6] R. Courant and D. Hilbert, Methods of mathematical physics, vol. 1, Interscience, New York, 1953

[7] W. Purkert and J. vom Scheidt, Zur approximativen Lösung des Mittelungsproblems für die Eigenwerte stochastischer Differentialoperatoren, ZAMM, 57, 515-525 (1977)

[8] W. Purkert and J. vom Scheidt, Eine Störungsrechnung für die Eigenwerte und Eigenvektoren zufälliger Matrizen, Beiträge Zur Analysis, 11 (1978), 113-135

[9] W. Purkert and J. vom Scheidt, Ein Grenzverteilungssatz für stochastische Eigenwertprobleme, ZAMM, 59 (1979), 611-623

[10] W. Purkert and J. vom Scheidt, Stochastic eigenvalue problems for differential equations, Rep. Math. Phys., 15 (1979), 205-227

[11]J. vom Scheidt and W. Purkert, Limit theorems for solutions of stochastic differential equation problems, Int. J. Math. and Math. Sci., 3 (1980), 113-149 